\title{
Performance of combined constellation shaping and bit interleaved coded modulation with iterative decoding (BICM-ID)
}

\author{
T. Arafa, W. Sauer-Greff, and R. Urbansky \\ University of Kaiserslautern, Communications Engineering, Gottlieb-Daimler-Str., 67653 Kaiserslautern, Germany
}

\begin{abstract}
The increasing demand of achieving high data rates in modern communication systems requires highly efficient bandwidth utilization. For this purpose, multilevel modulation schemes are used in association with forward error correction (FEC) codes in order to approach the channel capacity. However, there is a gap between the capacity of a uniform signal constellation and the Shannon unconstrained capacity. This gap can be reduced by applying constellation shaping. The task of shaping is to modify a uniform distributed signal constellation towards a Gaussian like distribution. In this paper, we investigate different approaches to combine the constellation shaping with a bit interleaved coded modulation with iterative decoding (BICM-ID) system. Simulation results show that this combination can offer a shaping gain up to $0.6 \mathrm{~dB}$.
\end{abstract}

\section{Introduction}

Since the capacity of the additive white Gaussian noise (AWGN) channel was found by Shannon, most research in information theory is interested in designing a communication system that operates close to Shannon's limit. The channel capacity is the maximum mutual information between channel input and output. The capacity of the AWGN channel for transmitted signals with Gaussian distribution is called as Shannon limit and it can be expressed with Shannon's formula (Shannon, 1948): $\quad C_{2-\operatorname{dim}}=\log _{2}\left(1+\frac{E_{s}}{N_{o}}\right)$ $\mathrm{bit} / \mathrm{s} / \mathrm{Hz}$. Figure 1 shows the AWGN channel capacities with equiprobable $M$-QAM, for $M=4,16, \ldots, 1024$, compared with the Shannon limit. We observe from Fig. 1 that in the low SNR regime $(<5 \mathrm{~dB})$, the reduction in capacity due to using uniform signal constellation is negligible. Thus, in this case the binary coding techniques can approach the capac-

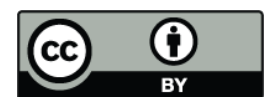

Correspondence to: T. Arafa (arafa@eit.uni-kl.de) ity limit. On the other hand, high order modulation schemes like $M$-QAM $(M>4)$ have to be used for good channel conditions in order to increase the spectral efficiency. However, the use of equiprobable signal constellations leads to a loss of $\pi e / 6(1.53 \mathrm{~dB})$ due to using a uniform distribution rather than a Gaussian distribution over the signal set (Forney and Ungerboeck, 1998). Therefore, the use of powerful channel coding with uniform constellation signal is not enough to achieve the capacity limit. The constellation shaping technique that produces a Gaussian-like distribution over constellation points has also to be used to close the gap of $1.53 \mathrm{~dB}$.

Higher level modulation schemes such as $M$-QAM can be used to achieve spectral efficient high data rate transmission over wireless, wireline, and optical links without increasing bandwidth. However, an increased density of the symbol constellation decreases its robustness against noise, which results in a high BER of the demapper. Therefore, the use of high order modulation schemes strongly requires channel coding to guarantee low BER at high bandwidth efficiency. Since an erroneous detected symbol may result in a burst of bit errors and channel codes are often sensitive to burst error, a permutation of the bit sequence has to be introduced between coding and mapping, so-called interleaving. The concatenation of channel coding, interleaving and modulation is denoted as a bit interleaved coded modulation (BICM). Iteratively demapping and decoding BICM (BICM-ID) can improve the performance by exchanging the soft information between soft-demapper and channel decoder.

Constellation shaping is a method to shape an $M$-QAM signal constellation to follow the Gaussian distribution. Actually, two techniques for constellation shaping have been mainly proposed in the literature. The first approach is to transmit the constellation points with unequal probability (Fischer, 2002; Khoo et al., 2006). The other is based on optimizing of the constellation points, i.e. using equiprobable signal constellations with unequal spacing (Sommer and Fettweis, 2000; Fischer, 2002; Djordjevic et al., 2010).

Published by Copernicus Publications on behalf of the URSI Landesausschuss in der Bundesrepublik Deutschland e.V. 


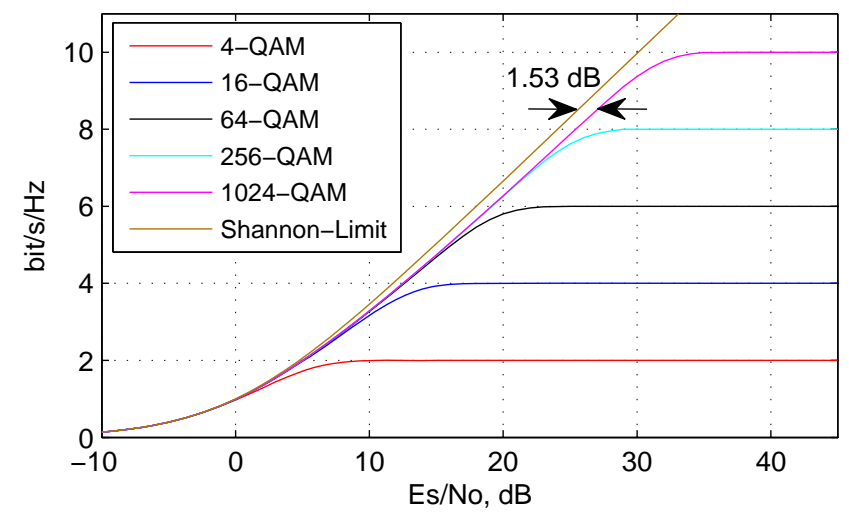

Fig. 1. The ideal AWGN channel capacities of equiprobable $M$ QAM signals compared with that of Gaussian signal.

The remainder of this paper is organized as follows. The concept of BICM-ID is briefly introduced in Sect. 2. In Sect. 3, the constellation shaping with different approaches including unequal probability and unequal spacing constellation points are presented. Since all BICM-ID systems suffer from an error floor, the inner precoding that can remove the error floor without adding extra redundancy bits is applied to the system in Sect. 4. Then, the chain of FEC coding, inner precoding and mapping will be optimized by means of extrinsic information transfer (EXIT) chart analysis. Simulation results and discussions are provided in Sect. 5. Finally, conclusions are drawn in Sect. 6.

\section{BICM-ID concept}

It is well accepted that the concatenation of code systems with iterative decoding can approach the channel capacity. The BICM-ID scheme is a special case of serial concatenated code systems where the turbo principle is applied between the inner decoder, i.e. the soft-demapper and the outer channel decoder (ten Brink et al., 1998).

The block diagram of the BICM-ID scheme is depicted in Fig. 2. At the transmitter, the sequence of $k$ data bits is firstly encoded into a codeword of $n$ bits by a recursive systematic convolutional (RSC) encoder where the code rate is $R=\frac{k}{n}$, which can be obtained, e.g., by puncturing. The encoded bits are randomly interleaved by a bit-wise interleaver. After interleaving, a group of $m$ coded bits is mapped onto one complex symbol of $M=2^{m}$ according to a mapping scheme. Therefore, the spectral efficiency of this system is given by

$\eta=R \cdot m \quad \mathrm{bit} / \mathrm{s} / \mathrm{Hz}$.

The originated complex symbols are corrupted by noise through the AWGN channel, so the received symbols are $\underline{r}=y+\underline{n}$ where $\underline{n}$ is a complex circular symmetric Gaussian noise with zero mean and variance $N_{o} / 2$ in each dimension. At the receiver, and based on channel observations $\underline{r}$

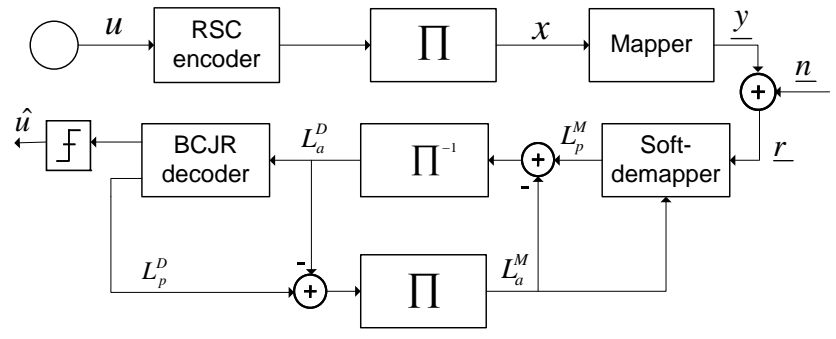

Fig. 2. BICM-ID system model.

and a-priori information of unmapped bits $L_{\mathrm{a}}^{M}$ (at first iteration $L_{\mathrm{a}}^{M}=0$ ), the soft-demapper calculates the a-posteriori log-likelihood ratio (LLR) for each of the $m$ coded bits per symbol by using (ten Brink et al., 1998)

$$
\begin{array}{r}
L_{p}^{M}\left(x_{k} \mid \underline{r}\right)=L_{a}^{M}\left(x_{k}\right) \\
+\ln \frac{\sum_{y \in \chi_{k}^{1}} \exp \left[-\frac{\underline{\underline{r}-\left.\underline{y}\right|^{2}}}{N_{o}}+\sum_{j=1, j \neq k}^{m} \mu_{j}^{-1}(\underline{y}) L_{\mathrm{a}}^{M}\left(x_{j}\right)\right]}{\sum_{y \in \chi_{k}^{0}} \exp \left[-\frac{\mid \underline{\underline{r}-\left.\underline{y}\right|^{2}}}{N_{o}}+\sum_{j=1, j \neq k}^{m} \mu_{j}^{-1}(\underline{y}) L_{\mathrm{a}}^{M}\left(x_{j}\right)\right]},
\end{array}
$$

where $\mu_{j}^{-1}(\underline{y})$ gives the $j$-th bit of the symbol $\underline{y}$ while $\chi_{k}^{1}, \chi_{k}^{0}$ is the set of symbols having the $k$-th bit set to 1 and 0 , respectively. Then, the extrinsic information of the soft-demapper $L_{\mathrm{e}}^{M}=L_{\mathrm{p}}^{M}-L_{\mathrm{a}}^{M}$ is deinterleaved to become the a-priori input $L_{\mathrm{a}}^{D}$ to the outer soft-in/soft-out decoder, which gives the a-posteriori value $L_{\mathrm{p}}^{D}$ based on the BCJR algorithm (Hagenauer, 1997). The extrinsic information of the outer decoder $L_{\mathrm{e}}^{D}=L_{\mathrm{p}}^{D}-L_{\mathrm{a}}^{D}$ is passed through the interleaver and fed back as a-priori information $L_{\mathrm{a}}^{M}$ to the soft-demapper for the next iteration.

As we have seen, only extrinsic information is exchanged between inner soft-demapper and outer decoder at the iteratively decoded BICM system. Therefore, the performance of this system can be analyzed and predicted by using the EXIT chart (ten Brink, 1999). It is an efficient tool to visualize the convergence behaviour and the BER floor of a concatenated coding system. Moreover, it graphically shows the relationship between the input mutual information against the output mutual information from the soft-demapper and decoder which help us to select the coding and mapping schemes that match each other.

In this context, different EXIT characteristics of mapping can be simply constructed by an irregular modulation (modulation doping) that applies Gray as well as anti-Gray mapping according to the doping ratio

$$
\alpha=\frac{N_{\text {anti-Gray }}}{N_{\text {anti-Gray }}+N_{\text {Gray }}}
$$

within the same transmitted block (Szczecinski et al., 2005). 


\section{Constellation shaping}

Constellation shaping is used to obtain a shaping gain over uniform signaling. The ultimate shaping gain for high data rates is $1.53 \mathrm{~dB}$. Essentially, two approaches have been proposed for signal shaping. The first approach is to shape the signal constellation to have a non-uniform probability distribution. With the other approach, the signal constellation is non-uniformly spaced.

\subsection{Shaping with non-uniform probability distribution over constellation points}

The basic idea of this approach is to transmit the symbols with unequal probability. In this case, the points with low energy, i.e. the points near the centre of the constellation are more frequently transmitted than the points with high energy. For example, Khoo et al. (2006) proposed to divide the 16-QAM constellation into three sub-constellations with different power levels. The first sub-constellation contains four points with lowest energy, the eight points with medium energy forms the second sub-constellation and the third has four points with highest energy. Although every four bits are mapped onto one symbol, the first two bits can also be used to select one of these sub-constellations as follows: if we have 00 the first sub-constellation is chosen, 01 or 10 leads to the second and the third is selected for incoming bits 11. As we can see, we can obtain unequal probability over symbols if the first two bits fulfill this requirement $P_{r}(0)>P_{r}(1)$. This can be achieved by modifying the basic BICM-ID model as depicted in Fig. 3. The encoded sequence by a rate- $R_{c}$ RSC encoder is divided into four sequences by a serial-to-parallel $(\mathrm{S} / \mathrm{P})$ converter. Each sequence of the first and second composed $K$ bits is interleaved to $m_{1,2}$ and then broken into $L$ successive $K_{\mathrm{s}}$-bit blocks, where $K=L \cdot K_{\mathrm{s}}$. These $L$ successive blocks are serially fed to a rate- $R_{\mathrm{S}}$ shaping encoder (S-ENC) to generates $L$ successive $N_{\mathrm{s}}$-bit blocks, which result in $N$-bit sequences $s_{1,2}$, where $R_{\mathrm{S}}=\frac{K_{\mathrm{S}}}{N_{\mathrm{s}}}$. The shaping encoder is designed so that the probability of a zero at the sequences $s_{1,2}$ is maximized. Then, these two $N$-bit sequences are interleaved to $x_{1,2}$ and passed to the mapper. Each of the third and fourth sequence already includes $N$ bits and they are also interleaved to $x_{3,4}$ and passed to mapper. Thus, there are four $N$-bit sequences at the input of the mapper to generate $N$ symbols, which fulfill the unequal probability distribution. This transmission system is defined with spectral efficiency

$\eta=2 R_{c}\left(R_{s}+1\right) \quad \mathrm{bit} / \mathrm{s} / \mathrm{Hz}$.

At the receiver, the third and fourth sequences are directly processed between soft-demapper and channel decoder. A shaping decoder has to be used for the first and second sequences. It accepts $L$ blocks of a-priori information for info bits $L_{\mathrm{a}}^{S}\left(m_{1}(k), m_{2}(k)\right), k=1, \ldots, K_{\mathrm{S}}$ and for code bits $L_{\mathrm{a}}^{S}\left(s_{1}(n), s_{2}(n)\right), n=1, \ldots, N_{\mathrm{s}}$ which are generated from the

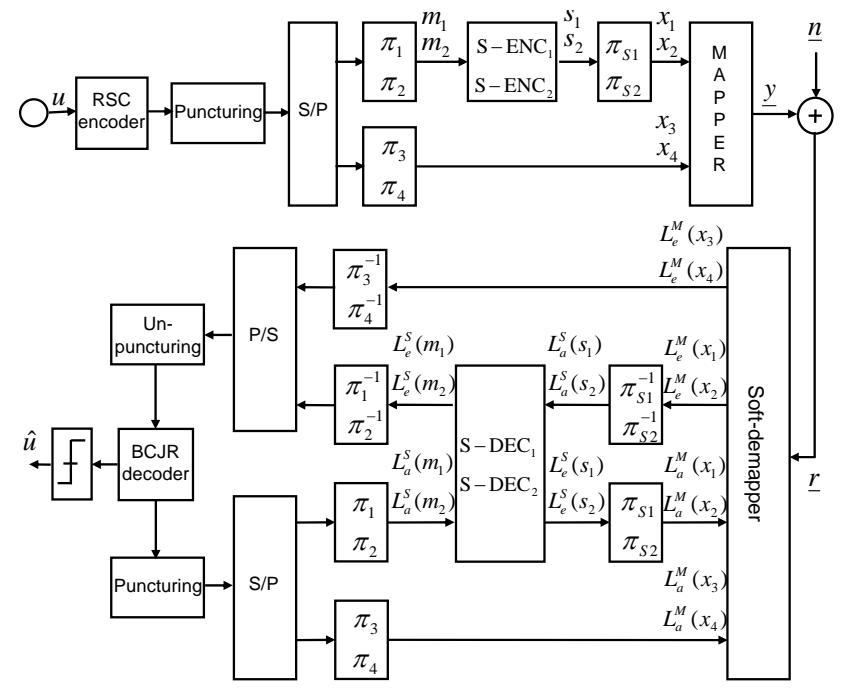

Fig. 3. BICM-ID system to shape 16-QAM with a shaping coding.

RSC decoder and soft-demapper, respectively, and calculates using these values the $L$ blocks of extrinsic information for info bits $L_{\mathrm{e}}^{S}\left(m_{1}(k), m_{2}(k)\right)$ and for code bits $L_{\mathrm{e}}^{S}\left(s_{1}(n), s_{2}(n)\right)$ which are passed to the RSC decoder and soft-demapper, respectively. Thus, the shaping decoder calculates the extrinsic information of both info bits and code bits based on the maximum a-posteriori algorithm (Khoo et al., 2006)

$L_{\mathrm{e}}^{S}(m(k))=\ln \frac{\sum_{M \in \Omega_{k}^{1}} \exp (A)}{\sum_{M \in \Omega_{k}^{0}} \exp (A)}$,

where

$A=\sum_{l=1, l \neq k}^{K_{\mathrm{S}}} t_{l} \cdot L_{\mathrm{a}}^{S}(m(l))+\sum_{n=1}^{N_{\mathrm{s}}} t_{n} \cdot\left(L_{\mathrm{a}}^{S}(s(n))-L_{n}\right)$,

$\Omega_{k}^{1}, \Omega_{k}^{0}$ is the set of all possible blocks $M$ of info having the $k$-th bit set to 1 and 0 , respectively, $t_{l}$ is the value of the $l$-th bit in the block $M$ under consideration, $t_{n}$ is the value of the $n$-th bit in the codeword $S$ associated to this particular block, $L_{n}=\ln \frac{P_{r}\{s(n)=0\}}{P_{r}\{s(n)=1\}}$ is the a-priori knowledge of bit $s(n)$ before the first iteration starts. Analogously

$L_{\mathrm{e}}^{S}(s(n))=\ln \frac{\sum_{S \in \Delta_{n}^{1}} \exp (B)}{\sum_{S \in \Delta_{n}^{0}} \exp (B)}$,

where

$B=\sum_{k=1}^{K_{\mathrm{s}}} t_{k} \cdot L_{\mathrm{a}}^{S}(m(k))+\sum_{l=1, l \neq n}^{N_{S}} t_{l} \cdot\left(L_{\mathrm{a}}^{S}(s(l))-L_{l}\right)$,

$\Delta_{n}^{1}, \Delta_{n}^{0}$ is the set of all possible codewords $S$ having the $n$-th bit set to 1 and 0 , respectively, $t_{l}$ is the value of the $l$-th bit 

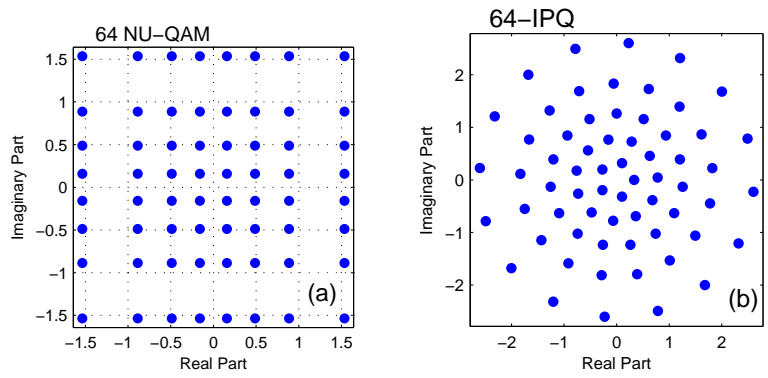

Fig. 4. Signal constellations of (a) 64-NU-QAM and (b) 64-IPQ.

in the codeword $S$ under consideration, and $t_{k}$ is the value of the $k$-th bit in the block $M$ associated to this particular codeword.

\subsection{Shaping with non-uniform spacing of constellation points}

It was proved in Sommer and Fettweis (2000), Fischer (2002), and Djordjevic et al. (2010) that by using equally likely signal points with unequal spacing the capacity limit can also be approached. In this method, the shaping gain can be obtained without any additional computational effort.

For optimizing the rectangular $M$-QAM constellation points, each dimension (i.e. $N$-ASK with $N=\sqrt{M}$ ) is optimized as follows. The probability of each $N$-ASK point is $1 / N$, but the coordinates $x_{i}, i=1,2, \ldots, N$, of the points are chosen appropriately. Therefore, the Gaussian cumulative distribution function $(\mathrm{CDF})$, which has value between zero and one, is partitioned into $N$ equal parts with $N+1$ boundaries $\frac{i}{N}, i=0,1, \ldots, N$. The coordinates $x_{i}$ of the signal points are then given as the centroids of these parts $\frac{\frac{i}{N}+\frac{i-1}{N}}{2}=\frac{2 i-1}{2 N}$, namely,

$x_{i}=\mathrm{CDF}^{-1}\left(\frac{2 i-1}{2 N}\right)=\sqrt{2} \operatorname{erf}^{-1}\left(\frac{2 i-1-N}{N}\right)$,

where $i=1,2, \ldots, N$. For example, Fig. 4a shows the nonuniform QAM signal constellation with 64 points (64-NUQAM) while the channel capacity for this constellation is depicted in Fig. 5. We can see that the 64-NU-QAM constellation is close to the optimum and outperforms the classical 64-QAM if the capacity is less than $5 \mathrm{bit} / \mathrm{s} / \mathrm{Hz}$.

On the other hand, the two dimensions of the circular QAM constellation can be jointly optimized. The optimized complex symbols have to follow a complex Gaussian distribution. The amplitude and phase of a complex Gaussian random variable are independent and conform a Rayleigh and a uniform distribution, respectively. By using this idea, the optimal circular signal constellation is generated based on an iterative polar quantization IPQ procedure (Djordjevic et al., 2010). In IPQ, the constellation points are distributed over the circles of radius determined by the Rayleigh distribution. Therefore, the circular $M$-QAM constellation is optimized

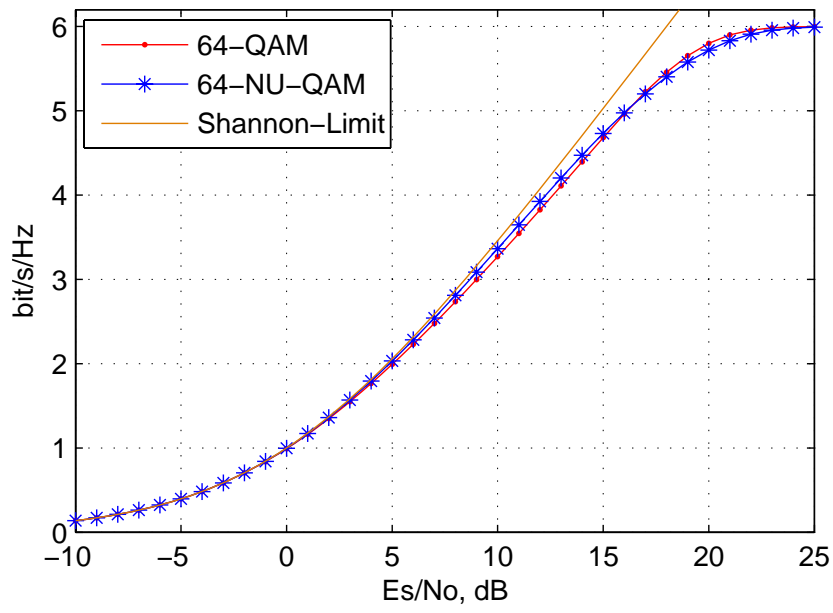

Fig. 5. Channel capacity of 64-NU-QAM versus 64-QAM.

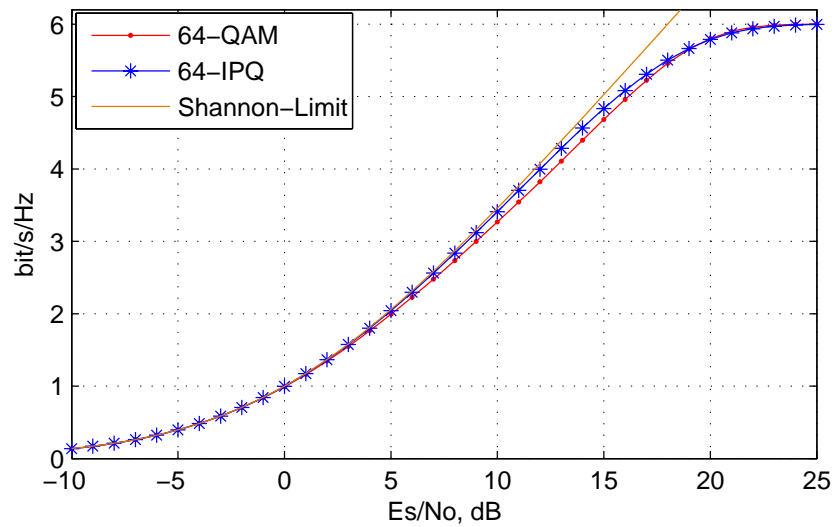

Fig. 6. Channel capacity of 64-IPQ versus 64-QAM.

to get an $M$-IPQ constellation based on the minimum mean square quantization error (Djordjevic et al., 2010). The optimum number of points $L_{i}$ per $i$-th circle with radius $m_{i}$ is determined by (Djordjevic et al., 2010)

$L_{i}=\frac{\sqrt[3]{m_{i}^{2} \int_{r_{i}}^{r_{i+1}} p(r) \mathrm{d} r}}{\sum_{i=2}^{L_{r}} \frac{1}{M} \sqrt[3]{m_{i}^{2} \int_{r_{i}}^{r_{i+1}} p(r) \mathrm{d} r}}, \quad i=1,2, \ldots, L_{r}$,

where $L_{r}$ is the number of circles in the constellation and $p(r)$ is the Rayleigh distribution function. The radius of the $i$-th circle is determined by

$m_{i}=\frac{2 \sin \left(\Delta \theta_{i} / 2\right) \int_{r_{i}}^{r_{i+1}} r p(r) \mathrm{d} r}{\Delta \theta_{i} \int_{r_{i}}^{r_{i+1}} p(r) \mathrm{d} r}, \quad \Delta \theta_{i}=\frac{2 \pi}{L_{i}}$.

The limits of integration in Eqs. (10) and (11) are determined by

$r_{i}=\frac{\pi\left(m_{i}^{2}-m_{i-1}^{2}\right)}{2\left[m_{i} L_{i} \sin \left(\Delta \theta_{i} / 2\right)-m_{i-1} L_{i-1} \sin \left(\Delta \theta_{i-1} / 2\right)\right]}$. 


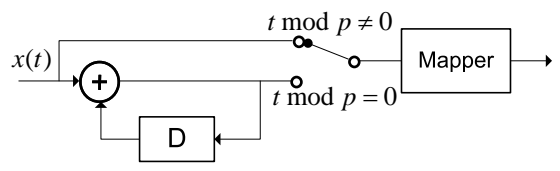

Fig. 7. Additional inner rate-one RSC code with doping.

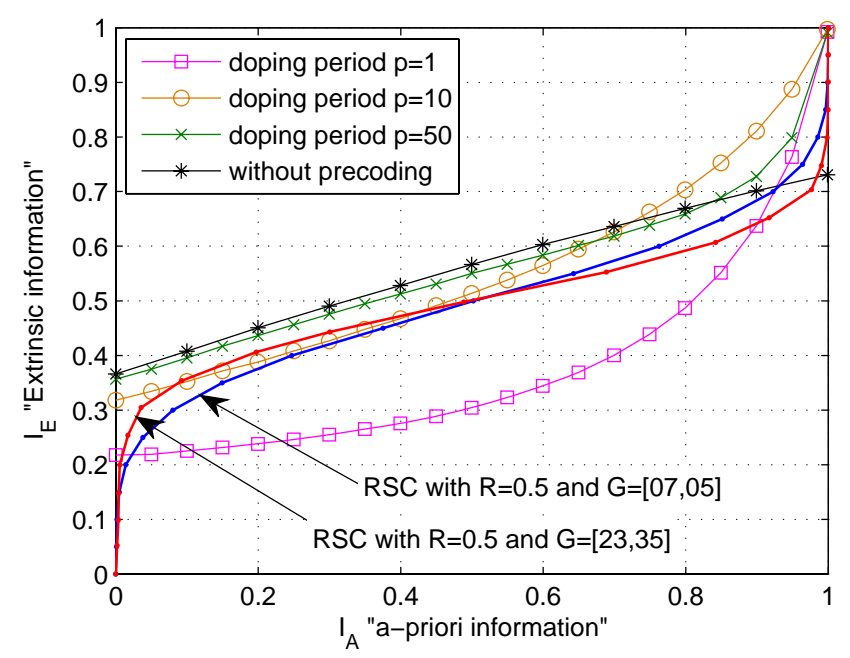

Fig. 8. EXIT chart of 64-IPQ mapping with different doping periods at $E_{b} / N_{o}=5.25 \mathrm{~dB}$.

In Fig. 4b we depict the 64-IPQ constellation. The corresponding channel capacity is shown in Fig. 6. As we can observe from this figure, the 64-IPQ constellation is very close to the Shannon limit and outperforms significantly the classical 64-QAM for low and medium SNRs.

\section{Error floor problem at BICM-ID systems}

In general, all BICM-ID systems suffer highly from an error floor. The reason of this phenomenon can be explained using an EXIT chart. The EXIT-characteristic of soft-demappers mostly does not reach to the point $(1,1)$, i.e. the intersection between the EXIT curves of soft-demapper and decoder is at $I_{\mathrm{e}}^{M}<1$ for $I_{\mathrm{a}}^{M} \approx 1$. This means that even for ideal a-priori information, the obtained extrinsic information is not perfect and some bit errors remain.

This problem can be solved if the tail of the soft-demapper curve (for $I_{\mathrm{a}}^{M}>0.5$ ) is bent up to reach point $(1,1)$ without lowering it for $I_{\mathrm{a}}^{M}<0.5$. This can be achieved by using a doping precoder (Pfletschinger and Sanzi, 2004). By this approach, a rate-1 RSC is inserted between interleaver and mapper as depicted in Fig. 7. The output of the inner encoder is doped where every $p$-th info bit is replaced by a coded bit and thus the rate is maintained. This scheme is defined with a doping period $p$, which has to be chosen rather high to achieve the mentioned requirement as shown in Fig. 8.

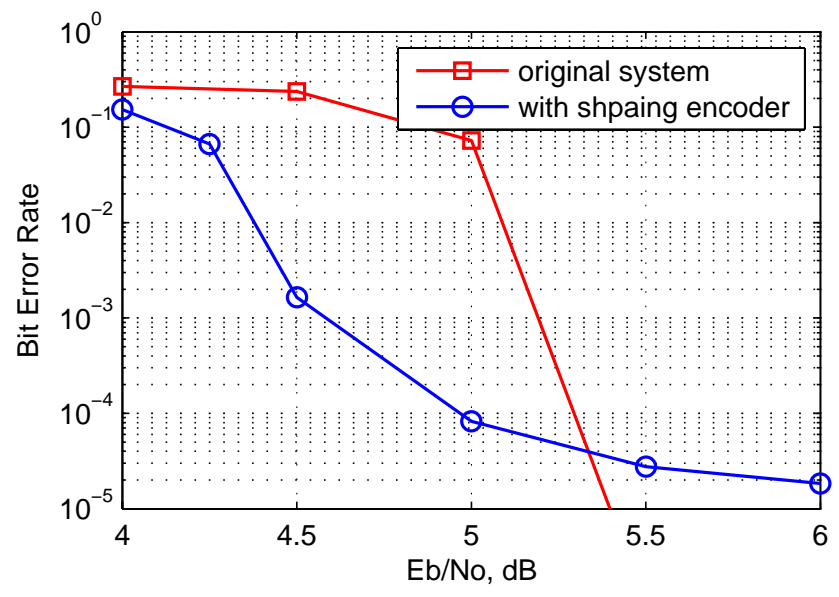

Fig. 9. Performance of BICM-ID with and without shaping encoder after 10 iterations.

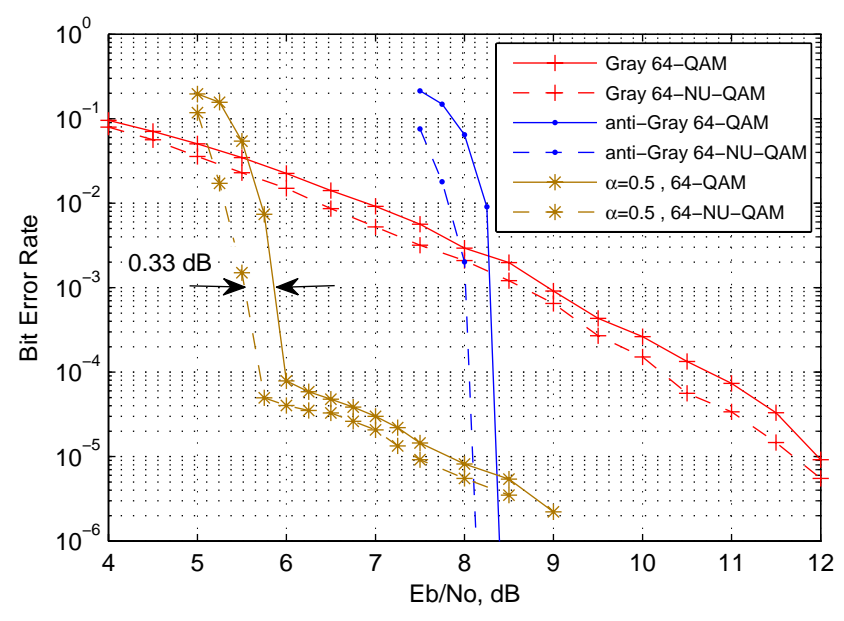

Fig. 10. BER of BICM-ID with different 64-QAM mapping schemes after 14 iterations.

\section{Simulation results}

Figure 9 shows the BER performance of the BICM-ID systems applying an anti-Gray 16-QAM mapping scheme without and with a shaping encoder at spectral efficiency $\eta=$ $2 \mathrm{bit} / \mathrm{s} / \mathrm{Hz}$. To ensure that both schemes operate at the same spectral efficiency, we used a rate $1 / 2$ RSC code with generator polynomials in octal form $G=[23,35]$ for the original system and a rate $2 / 3$ punctured RSC code with a rate $1 / 2$ shaping encoder for the system with shaping coding. The shaping encoder receives the info bits blockwise with $K_{\mathrm{s}}=7$ and generates the corresponding codeword with $N_{\mathrm{s}}=14$ which results in moderate complexity. As can be seen from Fig. 9, the system with a shaping encoder is $0.61 \mathrm{~dB}$ better than the original system at $\mathrm{BER}=10^{-3}$. However, this scheme shows an error floor occurring at $\mathrm{BER} \approx 10^{-5}$ due to the relatively poor error capabilities of a punctured rate $2 / 3$ RSC code. 


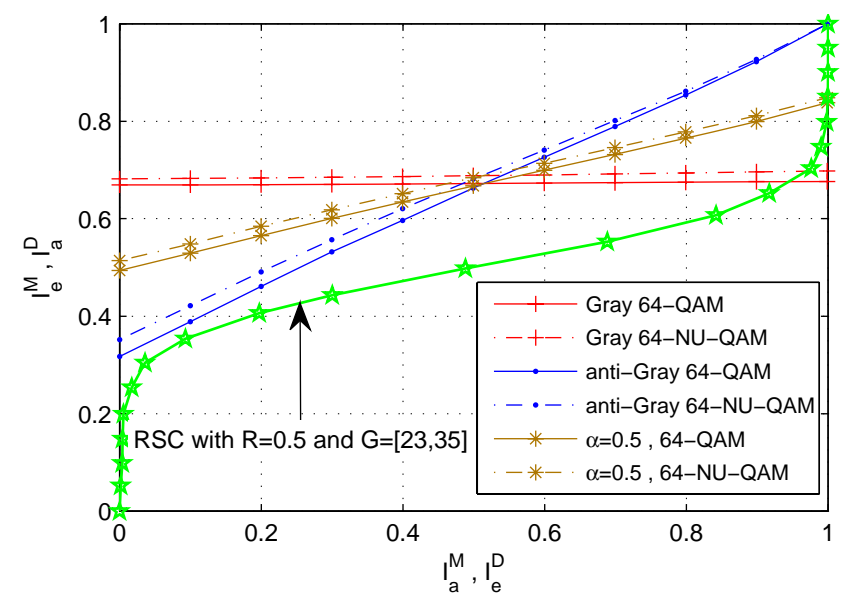

Fig. 11. EXIT chart of different 64-QAM mapping schemes at $E_{b} / N_{o}=8 \mathrm{~dB}$.
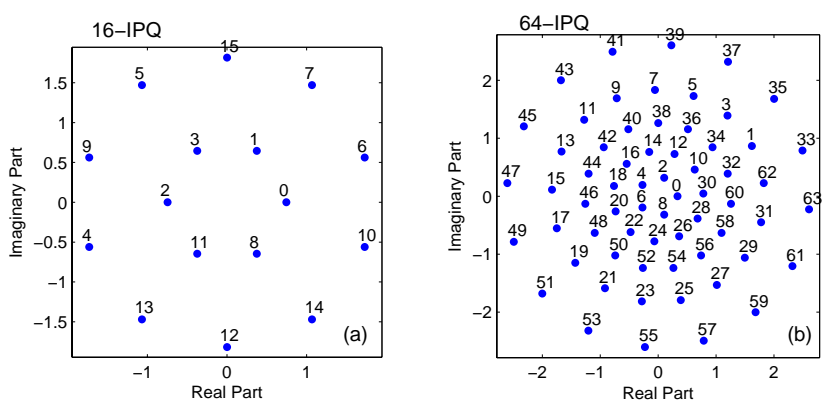

Fig. 12. Proposed mapping schemes for constellations of (a) 16IPQ; (b) 64-IPQ.

The performance of a BICM-ID system for both uniform (64-QAM) and non-uniform (64-UN-QAM) spacing of constellation points with different mapping schemes, Gray, antiGray and modulation doping $(\alpha=0.5)$ is depicted in Fig. 10. The corresponding EXIT chart is shown in Fig. 11. The simulation results show that the optimized rectangular constellation 64-NU-QAM offers an improvement of $0.33 \mathrm{~dB}$ for all mapping schemes. On the other hand, the modulation doping $(\alpha=0.5)$ combines the benefits of both Gray and anti-Gray mapping and outperforms the both, where it reaches to the turbo cliff at low SNR but suffer then from error floor as explained in Sect. 4. These results match to the EXIT chart where the soft-demapper curve is slightly shifted towards the top by using 64-NU-QAM for Gray, anti-Gray and modulation doping which means an increase in the capacity.

Finally, the performance of BICM-ID systems with 16IPQ and 64-IPQ mapping are evaluated. Since constructing Gray and anti-Gray mapping schemes for arbitrary $M$ IPQ constellations is not easy, we try to construct the mapping schemes for 16-IPQ and 64-IPQ so that the proposed schemes match the outer RSC coding with a memory size of 4. The proposed mapping schemes for 16-IPQ and 64-IPQ

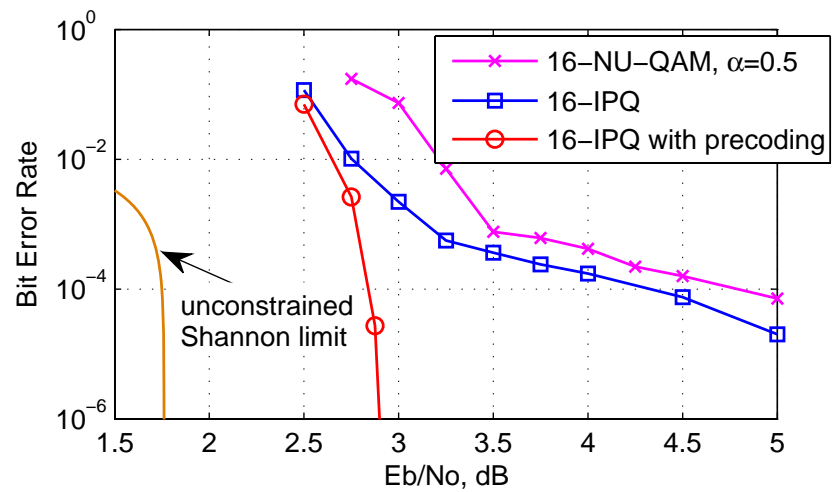

Fig. 13. BER of BICM-ID without and with precoding for 16-IPQ mapping after 25 iterations.

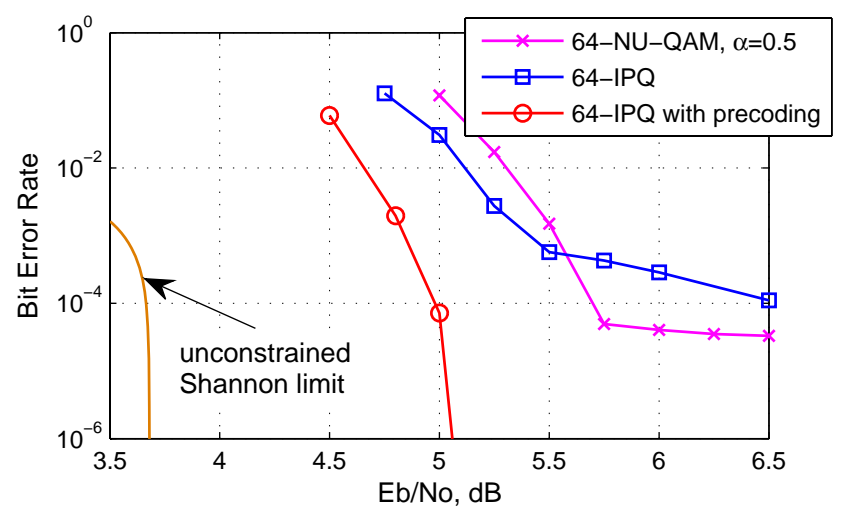

Fig. 14. BER of BICM-ID without and with precoding for 64-IPQ mapping after 25 iterations.

are depicted in Fig. 12. The use of 16-IPQ and 64-IPQ offers an improvement of $0.32 \mathrm{~dB}$ and $0.12 \mathrm{~dB}$ at $\mathrm{BER}=10^{-3}$ compared with 16-NU-QAM and 64-NU-QAM with doping ratio $\alpha=0.5$, respectively.

On the other hand, 16-IPQ and 64-IPQ mapping schemes, as expected, suffer from an error floor. It can be removed by using precoding with a doping period $p=50$. Moreover, by using precoding with selecting a memory-2 RSC encoder for matching the coding transfer curve to the new mapper curve with precoding as shown in Fig. 8, the performance can be improved as depicted in Fig. 13 and Fig. 14. The performance is $1.09 \mathrm{~dB}$ and $1.27 \mathrm{~dB}$ away from the unconstrained Shannon limit at BER $=10^{-3}$ for 2 and $3 \mathrm{bit} / \mathrm{s} / \mathrm{Hz}$, respectively, with moderate complexity.

\section{Conclusions}

In this contribution we presented the combined constellation shaping and BICM-ID system. A shaping gain of $0.6 \mathrm{~dB}$ can be obtained with unequal probability over constellation points by adding a shaping encoder which results in high complexity compared to the original system. On the other 
hand, the optimized unequal spacing of points for both rectangular and circular constellations achieves approximately the capacity and can be applied to a BICM-ID system without any increase in complexity.

\section{References}

Djordjevic, I. B., Batshon, H. G., Xu, L., and Wang, T.: Coded Polarization-Multiplexed Iterative Polar Modulation (PM-IPM) for Beyond $400 \mathrm{~Gb} / \mathrm{s}$ Serial Optical Transmission, Optical Fiber Communication Conference (OFC), San Diego, California, USA, 21-25 March 2010, OMK2, 2010.

Fischer, R. F. H.: Precoding and Signal Shaping for Digital Transmission, John Wiley \& Sons Inc., New York, USA, 2002.

Forney Jr., G. D. and Ungerboeck, G.: Modulation and Coding for Linear Gaussian Channels, IEEE Transactions Information Theory, 44, 2384-2415, 1998.

Hagenauer, J.: The Turbo Principle: Tutorial Introduction and State of the Arts, Symposium on Turbo Codes, Brest, France, September 1997, 1-11, 1997.

Khoo, B. K., Le Goff, S. Y., Sharif, B. S., and Tsimenidis, C. C.: Bit-Interleaved Coded Modulation with Iterative Decoding Using Constellation Shaping, IEEE Transactions on Communications, 54, 1517-1520, 2006.
Pfletschinger, S. and Sanzi, F.: Iterative Demapping for OFDM with Zero-Padding or Cyclic Prefix, IEEE International Conference on Communications, Paris, France, 20-24 June 2004, 842-846, 2004.

Shannon, C. E.: A Mathematical Theory of Communication, Bell System Technical Journal, 27, 379-423 and 623-656, 1948.

Sommer, D. and Fettweis, G. P.: Signal Shaping by Non-Uniform QAM for AWGN Channels and Applications Using Turbo Coding, International ITG Conference on Source and Channel Coding, Munich, Germany, 17-19 January 2000, 81-86, 2000.

Szczecinski, L., Chafnaji, H., and Hermosilla, C.: Modulation Doping for Iterative Demapping of Bit-Interleaved Coded Modulation, IEEE Communications Letters, 9, 1031-1033, 2005.

ten Brink, S.: Convergence of Iterative Decoding, IEEE Electronics Letters, 35, 1117-1119, 1999.

ten Brink, S., Speidel, J., and Yan, R.-H.: Iterative Demapping and Decoding for Multilevel Modulation, IEEE International Conference on Global Communications (GLOBECOM), Sydney, Australia, 8-12 November 1998, 579-584, 1998. 